\title{
ACRL members running for ALA Council
}

The following ACRL members are either nominated or petition candidates for ALA councilor in the spring 2011 election. ACRL members are encouraged to vote for these candidates to increase ACRL's voice in ALA affairs.

J. Douglas (Doug) Archer, Reference and Peace Studies Librarian, University of Notre Dame

Karl Bridges, Associate Professor, Information and Instruction Services, University of Vermont

Matthew P. Ciszek, Head Librarian, Penn State Shenango

Roberto Carlos Delgadillo, Humanities, Social Sciences, and Government Information Services Librarian, University of California-Davis

John DeSantis, Cataloging and Metadata Services Librarian, Dartmouth College

Jennifer Emanuel, Digital Services and Reference Librarian, University of Illinois Urbana-Champaign

Martin Garnar, Reference Services Librarian, Regis University

ShuYong Jiang, Librarian/Catalog Coordinator, University of Illinois Urbana-Champaign

Em Claire Knowles, Assistant Dean, Simmons College

Kate Kosturski, Independent Consultant, Outreach and Participation Services, JSTOR

Charles E. Kratz, Dean of Libraries, University of Scranton

LeRoy LaFleur, Head, Arlington Campus Library, George Mason University
Patricia Ann Loghry, Audio Visual Cataloger, University of Notre Dame

Dallas Jonathan Long, Head of Access Services, Illinois State University

Lawrence McCrank, Dean, Library and Instruction Services, Chicago State University

Courtney Mlinar, Academic Support Services/Reference Librarian, Nova Southeastern University

Toni Negro, Librarian, Universities at Shady Grove

Andrew K. Pace, Executive Director, Networked Library Services, OCLC

Mary Pagliero Popp, Research and Discovery Services Librarian, Indiana University-Bloomington

JP Porcaro, Virtual Services Librarian, New Jersey City University

Zora Sampson, Director, Karrmann Library, University of Wisconsin-Platteville

Julie Schneider, Library Director, University of Wisconsin-Madison

John C. Stachacz, Dean of Library Services, Wilkes University

James K. Teliha, Associate University Librarian for Public Services, Idaho State University

Holly Tomren, Head of Monograph, Electronic Resources and Metadata Cataloging, University of California-Irvine

Patricia A. Wand, Consultant 